\title{
Object Extraction Focused on Holding Action of Human Being
}

\author{
Tatsuki Ishijima ${ }^{\text {a }}$, Masaki Ishii ${ }^{\text {a,* }}$ \\ ${ }^{a}$ Akita Prefectural University, 84-4 Tsuchiya-Ebinokuchi, Yurihonjo city 015-0055, Japan \\ *Corresponding Author: ishii@akita-pu.ac.jp
}

\begin{abstract}
In recent years, robots have become a part of the household. To facilitate the greater integration of robots into human life, it has become necessary to develop highrecognition functions for robots. We believe that the usage of objects, i.e., the mutual relationship between a human and an object should be a feature of the human recognition function. In this study, we detect a basic interaction between a human and an object, and develop an object recognition method using an image and interaction (action) when the interaction occurs. The general flow of image recognition processing is as follows: feature extraction, learning, and recognition. There is a problem, however: it is difficult to extract features by occlusion and similar colors during feature extraction. In this paper, we focus on the interaction between a human and an object as a method of obtaining an effective image for feature extraction. We discuss a robust object extraction method using the joint position information of the human and depth information of similar colors and occlusion.
\end{abstract}

Keywords: object extraction, holding action.

\section{Introduction}

Recent years have seen considerable progress in the development of robots and in facilitating their coexistence with humans. To achieve the latter, it has become necessary to develop high-recognition functions such as object recognition. Object recognition by a computer in a complicated environment is difficult because it requires the extraction of an effective object characteristic to detect and recognize an object using only physical factors. On the other hand, humans obtain a lot more information through vision, and they also recognize objects by association and personal experience. This association is the interaction between a human and an object.
In this paper, an interaction refers to an interactive action, i.e., one that is a specific action of a human touching an object. For example, consider a pencil, which is a stick, lying on a desk. The action of "writing" occurs when a human holds the pencil and moves its tip in contact with a piece of paper; the human recognizes the stick to be a pencil. Therefore, a human's interaction with an object provides important information in recognizing the object. We believe that usage of an object, i.e., the mutual relationship between a human and an object, should be a feature of the human recognition function. In this study, we detect a basic interaction between a human and an object, and develop an object recognition method using an image and interaction (action) when interaction occurs. In this paper, we focus on the holding action of a human being, and discuss an object extraction method using joint position information and depth information.

\section{Conventional Method and Problems}

The general flow of image recognition processing is as follows: input data, feature extraction from images, learning, and recognition. In addition, images are used as input data in this case. Feature extraction and learning from the image, to be used as the input data, have a great influence on recognition ${ }^{(1)}$. Feature extraction is dependent on the input data. It is difficult to extract features in the image by using similar colors and occlusions that overlap the object and object. As for the image extracting feature, an image in which only a recognition object appears is appropriate. Therefore, it is necessary to extract only the recognition target. Object extraction by edge and color is used as a conventional method. However, it is difficult to extract an object by occlusion, and there are color problems in the conventional method when edges and colors are used.

In a prior study ${ }^{(2)}$, we performed object extraction not based on edges and colors but the joint position information 
of a human and depth information using an RGB-D sensor. This method can extract only an object, but occlusions owing the hand are difficult to extract after a holding action.

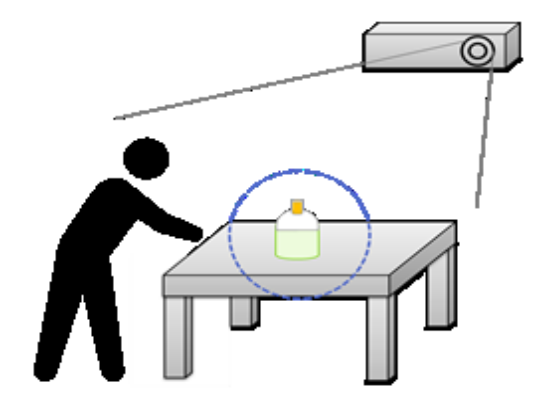

Fig.1. Overview of proposed method

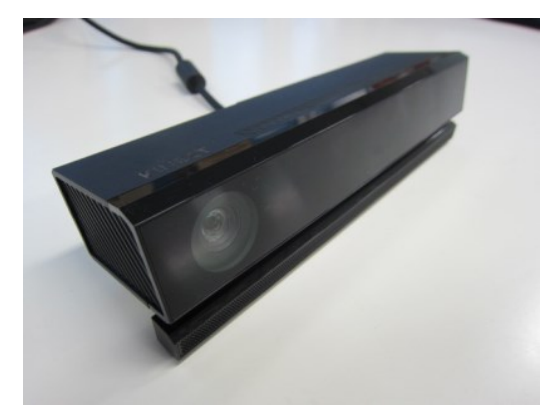

Fig.2. Appearance of the Kinect v2

Table 1. Kinect v2 specifications

\begin{tabular}{|c|c|c|}
\hline \multirow{2}{*}{\multicolumn{2}{|c|}{ Color }} & $1920 \times 1080$ pixel \\
\hline & & $30 \mathrm{fps}$ \\
\hline \multirow{2}{*}{\multicolumn{2}{|c|}{ Depth }} & $512 \times 424$ pixel \\
\hline & & $30 \mathrm{fps}$ \\
\hline \multicolumn{2}{|l|}{ Player } & 6 people \\
\hline \multicolumn{2}{|l|}{ Skeleton } & 6 people \\
\hline \multicolumn{2}{|l|}{ Joint } & 25 joint/person \\
\hline \multicolumn{2}{|c|}{ Range of Depth } & $0.5-8.0 \mathrm{~m}$ \\
\hline \multicolumn{2}{|c|}{ Range of Detection } & $0.5-4.5 \mathrm{~m}$ \\
\hline \multirow{2}{*}{ Angle Depth } & Horizontal & $70^{\circ}$ \\
\hline & Vertical & $60^{\circ}$ \\
\hline
\end{tabular}

Table 2. PC specifications

\begin{tabular}{|l|l|}
\hline OS & Windows 10 Home 64bit \\
\hline CPU & Intel ${ }^{\circledR}$ Core ${ }^{\mathrm{TM}}$ i7-5500U @ $2.40 \mathrm{GHz}$ \\
\hline Memory & $8 \mathrm{~GB}$ \\
\hline SDK & Kinect for Windows SDK v2 \\
\hline
\end{tabular}

\section{Proposal Method}

The action of "having" or "holding" is performed when a human touches an object. Therefore, it is expected that the object exists near the hand. However, occlusions resulting from the hand influence the following recognition processing when an object is extracted after an action. In this paper, we assumed that the target object exists near the hand. We focus on the action of "having" or "holding" just before the scene. We believe that we can acquire an image without occlusions owing the hand. This is effective for recognition by extracting an object just before an action. In this study, we performed an object extraction around the hand using an RGB-D sensor (Microsoft Corporation, Kinect for Windows v2). Fig. 1 and 2 show, respectively, an overview of the proposed method and the appearance of the Kinect v2. Table 1 and 2 list the specifications for the Kinect $\mathrm{v} 2$ and the PC that were used for our experiments.

The processing procedure for the object extraction method proposed in this paper is shown below. The resolution of the image is $512 \times 424$ pixels because we use a depth image.

I. Acquisition of the depth image

II. Eliminate the human area

III. Acquire the joint coordinates of the human

IV. Calculate the end of the hand coordinates by using hand and elbow coordinates

V. Acquire depth information at the hand coordinates

VI. Segment by threshold processing using the depth information mentioned above

VII. Acquire the Region Of Interest (ROI) around the end of the hand coordinates

VIII. Perform contour detection in the ROI

IX. Calculate an area in the biggest contour

$\mathrm{X}$. Extract the object by threshold processing using the area

At first, we used Kinect library for acquisition depth image. In addition, we detected a human at the same time, and eliminated the human area by painting white the pixels of detected human areas. Next, we acquired the skeleton coordinates of the human. We extracted hand and elbow coordinates from the skeleton coordinates. We calculated the end-of-hand coordinates $P\left(p_{x}, p_{y}\right)$ using equations (1) and (2). We identified $\left(x_{1}, y_{1}\right)$ as the elbow coordinates, $\left(x_{2}, y_{2}\right)$ as the 
hand coordinates, $l$ as the distance between the elbow coordinates and the hand coordinates, $n$ as the distance to the end of the hand and constant number. We decided the value of $n$ to be the same as $l$ in this paper. We can acquire the end coordinates using the distance from the elbow to the hand:

$$
\begin{aligned}
& p_{x}=\frac{-n x_{1}+(l+n) x_{2}}{l} \\
& p_{y}=\frac{-n y_{1}+(l+n) y_{2}}{l}
\end{aligned}
$$

Next, we acquired the depth information at the hand coordinates. We eliminated a region out of an anteroposterior constant range of the hand via threshold processing using the depth coordinates that we acquired. The threshold processing used equation (3). We identified $H_{\text {Depth }}$ as the depth information at the hand coordinates, and $X$ as the depth information at each set of coordinates.

$$
H_{\text {Depth }}-200<X<H_{\text {Depth }}+100
$$

We extracted the region around the end of the hand coordinates for images, and performed the abovementioned processing on those images. We used OpenCV from the image processing library for the extraction of region around the end of the hand. In addition, we used OpenCV for the following processing. The size of the extraction region was calculated based on the depth information from the camera to the hand. We identified one side of ROI 60-130 pixels of depth information from the camera that was located within 0.5-1.1 m. In addition, one side for every depth information $0.1 \mathrm{~m}$ by -10 pixels to increase one side based on depth information.

Next, we performed grayscale conversion, thresholding, and noise elimination in the extracted region. It was opening and closing process of morphological operation twice for noise elimination. Furthermore, we performed contour detection in the region and detected the biggest contour. We calculated the area within the biggest contour and extracted the target object by performing threshold processing. This was determined by the ratio of the area within the contour in the extraction region in threshold processing. Fig. 3 shows the result of each stage of image processing. In addition, Figs. 3(v) (vii) show the image before segmentation.

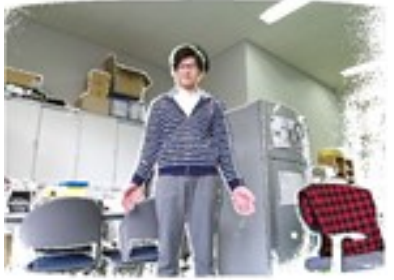

(i)

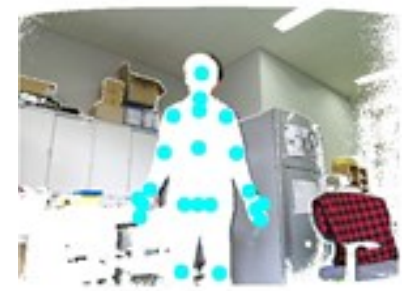

(iii)

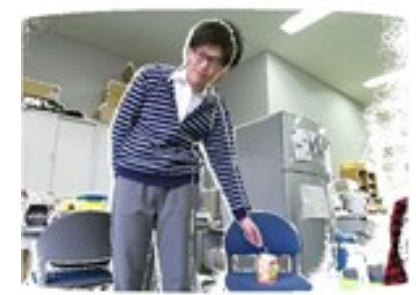

(v)

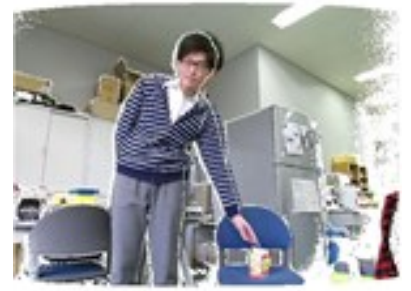

(vii)

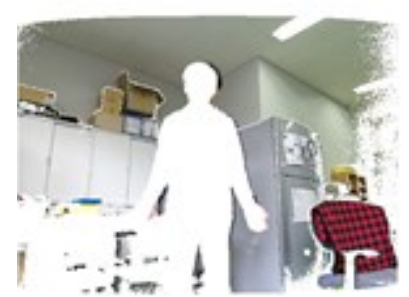

(ii)

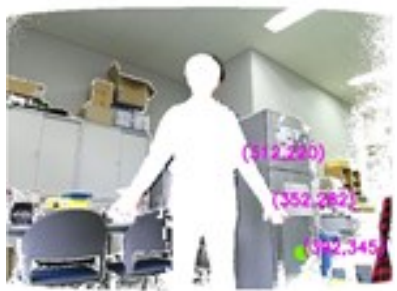

(iv)
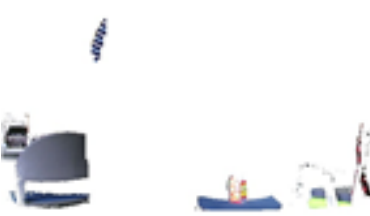

(vi)

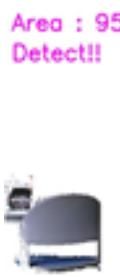

(viii)
Fig.3. Results of processing stages

\section{Experiment and Results}

The action of "having" or "holding" was assumed to be performed by the right hand. In addition, we performed experiments with a human standing and sitting. Fig. 4 shows the experimental situation at the standing state. Fig. 5 shows the result of object extraction at the standing state. In addition, Fig. 6 and 7 show the experimental situation for a human sitting state, and the result of object extraction at that time. We compared the result of object extraction at the standing state with sitting state. An object was extracted at both states except in direction (4). In addition, an object was not extracted at the sitting state in direction (4). We believe this occurred because the object region and human region did not completely overlap in direction (4) of the standing state. The other way, we 
believe that because could not segmentation with human and object in the cause of object region was completely included in a human region in the direction (4) of the sitting state. Therefore, we believe that object region was detected as a human region, and thus we could not extract the object by the being eliminated that region at the same time.

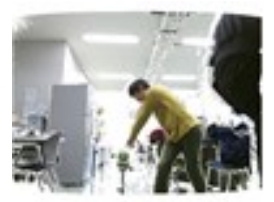

(1)

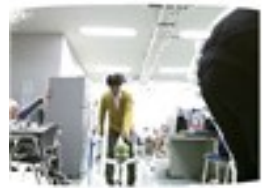

(4)

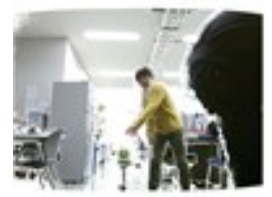

(2)

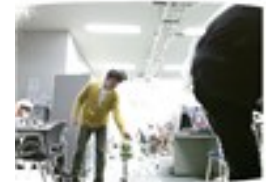

(5)

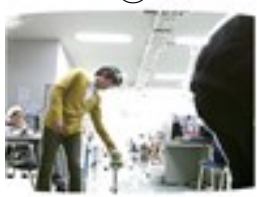

(7)

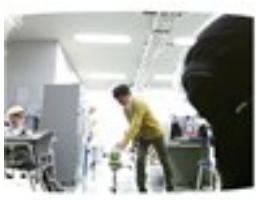

(3)

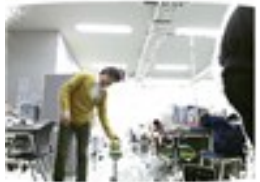

(6)

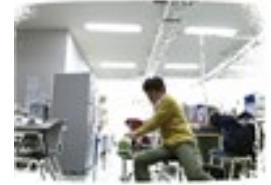

(1)

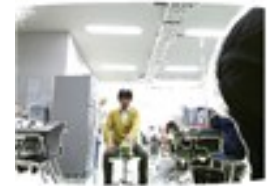

(4)

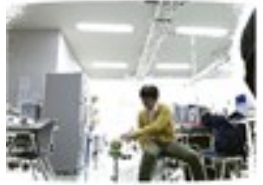

(2)

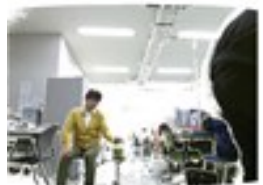

(5)

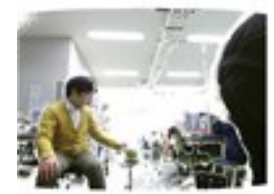

(7)

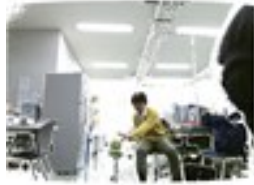

(3)

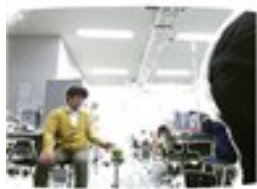

(6)
Fig. 6. Experiment of sitting state

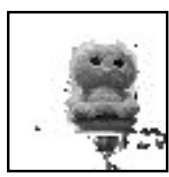

(1)

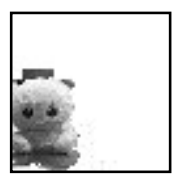

(5)

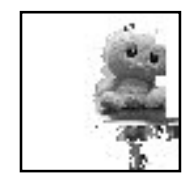

(2)

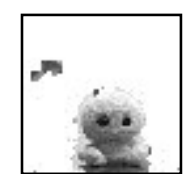

(6)

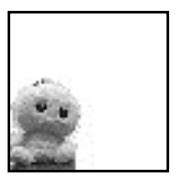

(3)

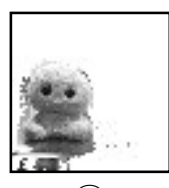

(7)
Fig. 5. Result of extraction at standing state

\section{Conclusion}

In this study, we assumed that a target object exists around the hand. We focused on the action of "having" or "holding" just before a scene. We tried to acquire an image without occlusions a resulting from the hand. This was effective for recognition by extracting an object just before the action. Object extraction was performed based on the joint position information of the human, and by depth information. As a result, we could acquire an image without
Fig. 7. Result of extraction at sitting state

\section{References}

(1) Daiki Hirose, Tsutomu Miyoshi : "Migrants recognition by image processing using distance sensor" Proceedings of The 75th National Convention of Information Processing Society of Japan, pp.579-580, 2013

(2) Tatsuki Ishijima, Masaki Ishii : "Object extraction focused on interaction with human being" 2016 National Convention Record of Institute of Electrical Engineers of japan, p63, 2016 